\title{
GENERALIZED PARTIALLY RELAXED PSEUDOMONOTONE VARIATIONAL INEQUALITIES AND GENERAL AUXILIARY PROBLEM PRINCIPLE
}

\author{
RAM U. VERMA
}

Received 30 April 2004; Accepted 29 August 2004

Let $T: K \rightarrow H$ be a nonlinear mapping from a nonempty closed invex subset $K$ of an infinite-dimensional Hilbert space $H$ into $H$. Let $f: K \rightarrow R$ be proper, invex, and lower semicontinuous on $K$ and let $h: K \rightarrow R$ be continuously Fréchet-differentiable on $K$ with $h^{\prime}$, the gradient of $h,(\eta, \alpha)$-strongly monotone, and $(\eta, \beta)$-Lipschitz continuous on $K$. Suppose that there exist an $x^{*} \in K$, and numbers $a>0, r \geq 0, \rho(a<\rho<\alpha)$ such that for all $t \in[0,1]$ and for all $x \in K^{*}$, the set $S^{*}$ defined by $S^{*}=\left\{(h, \eta): h^{\prime}\left(x^{*}+\right.\right.$ $\left.\left.t\left(x-x^{*}\right)\right)\left(x-x^{*}\right) \geq\left\langle h^{\prime}\left(x^{*}+t \eta\left(x, x^{*}\right)\right), \eta\left(x, x^{*}\right)\right\rangle\right\}$ is nonempty, where $K^{*}=\{x \in K$ : $\left.\left\|x-x^{*}\right\| \leq r\right\}$ and $\eta: K \times K \rightarrow H$ is $(\lambda)$-Lipschitz continuous with the following assumptions. (i) $\eta(x, y)+\eta(y, x)=0, \eta(x, y)=\eta(x, z)+\eta(z, y)$, and $\|\eta(x, y)\| \leq r$. (ii) For each fixed $y \in K$, map $x \rightarrow \eta(y, x)$ is sequentially continuous from the weak topology to the weak topology. If, in addition, $h^{\prime}$ is continuous from $H$ equipped with weak topology to $H$ equipped with strong topology, then the sequence $\left\{x^{k}\right\}$ generated by the general auxiliary problem principle converges to a solution $x^{*}$ of the variational inequality problem (VIP): $\left\langle T\left(x^{*}\right), \eta\left(x, x^{*}\right)\right\rangle+f(x)-f\left(x^{*}\right) \geq 0$ for all $x \in K$.

Copyright @ 2006 Hindawi Publishing Corporation. All rights reserved.

\section{Introduction}

A tremendous amount of work, applying the auxiliary problem principle in finite- as well as in infinite-dimensional Hilbert space settings, on the approximation-solvability of various classes of variational inequalities and complementarity problems, especially finitedimensional cases, has been carried out in recent years. During the course of these investigations, there has been a significant progress in developing more generalized classes of mappings in the context of new iterative algorithms. In this paper, we intend based on a general auxiliary problem principle to present the approximation-solvability of a class of variational inequality problems (VIP) involving partially relaxed pseudomonotone mappings along with some modified results on Fréchet-differentiable functions that play a pivotal role in the development of a general framework for the auxiliary problem principle. Results thus obtained generalize/complement investigations of Argyros and 
Verma [1], El Farouq [7], Verma [20], and others. For more details on general variational inequality problems and the auxiliary problem principle, we refer to [1-23].

Let $H$ be an infinite-dimensional real Hilbert space with the inner product $\langle x, y\rangle$ and norm $\|x\|$ for all $x, y \in H$. We consider the variational inequality problem (VIP) as follows: determine an element $x^{*} \in K$ such that

$$
\left\langle T\left(x^{*}\right), \eta\left(x, x^{*}\right)\right\rangle+f(x)-f\left(x^{*}\right) \geq 0 \quad \forall x \in K,
$$

where $K$ is a nonempty closed invex subset of $H$, and $\eta: K \times K \rightarrow H$ is any mapping with some additional conditions.

When $\eta\left(x, x^{*}\right)=x-x^{*}$, the VIP (1.1) reduces to the VIP: determine an element $x^{*} \in$ $K$ such that

$$
\left\langle T\left(x^{*}\right), x-x^{*}\right\rangle+f(x)-f\left(x^{*}\right) \geq 0 \quad \forall x \in K,
$$

where $K$ is a nonempty closed convex subset of $H$.

When $f=0$ in (1.2), it reduces to the following: find an element $x^{*} \in K$ such that

$$
\left\langle T\left(x^{*}\right), x-x^{*}\right\rangle \geq 0 \quad \forall x \in K \text {. }
$$

Now we recall the following auxiliary result for the approximation solvability of nonlinear variational inequality problems based on iterative procedures.

Lemma 1.1. For elements $u, v, w \in H$,

$$
\|u\|^{2}+\langle u, \eta(v, w)\rangle \geq-\frac{1}{4}\|\eta(v, w)\|^{2} .
$$

LeMma 1.2. For $u, v \in H$,

$$
\langle u, v\rangle=\frac{\|u+v\|^{2}-\|u\|^{2}-\|v\|^{2}}{2} .
$$

Now recall and in some cases upgrade the existing notions in the literature. Let $\eta: H \times H \rightarrow$ $H$ be any mapping.

Definition 1.3. A mapping $T: H \rightarrow H$ is called

(i) $(\eta)$-monotone if for each $x, y \in H$, there exists,

$$
\langle T(x)-T(y), \eta(x, y)\rangle \geq 0
$$

(ii) $(\eta, r)$-strongly monotone if there exists a positive constant $r$ such that

$$
\langle T(x)-T(y), \eta(x, y)\rangle \geq r\|x-y\|^{2} \quad \forall x, y \in H ;
$$

(iii) ( $r$ )-expansive if

$$
\|T(x)-T(y)\| \geq r\|\eta(x, y)\|
$$

(iv) expansive if $r=1$ in (iii), 
(v) $(\eta, \gamma)$-cocoercive if there exists a constant $\gamma>0$ such that

$$
\langle T(x)-T(y), \eta(x, y)\rangle \geq r\|T(x)-T(y)\|^{2} \quad \forall x, y \in H ;
$$

(vi) ( $\eta$ )-pseudomonotone if

$$
\langle T(y), \eta(x, y)\rangle \geq 0 \Longrightarrow\langle T(x), \eta(x, y)\rangle \geq 0 ;
$$

(vii) $(\eta, b)$-strongly pseudomonotone if

$$
\langle T(y), \eta(x, y)\rangle \geq 0 \Longrightarrow\langle T(x), \eta(x, y)\rangle \geq b\|x-y\|^{2} \quad \forall x, y \in H ;
$$

(viii) $(\eta, c)$-pseudococoercive if there exists a constant $c>0$ such that

$$
\langle T(y), \eta(x, y)\rangle \geq 0 \Longrightarrow\langle T(x), \eta(x, y)\rangle \geq c\|T(x)-T(y)\|^{2} \quad \forall x, y \in H ;
$$

(ix) ( $\eta$-quasimonotone if

$$
\langle T(y), \eta(x, y)\rangle>0 \Longrightarrow\langle T(x), \eta(x, y)\rangle \geq 0 \quad \forall x, y \in H
$$

(x) $(\eta, L)$-relaxed (also called weakly monotone) if there is a positive constant $L$ such that

$$
\langle T(x)-T(y), \eta(x, y)\rangle \geq(-L)\|x-y\|^{2} \quad \forall x, y \in H ;
$$

(xi) ( $\eta$-hemicontinuous if for all $x, y, w \in H$, the function

$$
t \in[0,1] \longrightarrow\langle T(y+t \eta(x, y)), w\rangle
$$

is continuous;

(xii) $(\eta, \beta)$-Lipschitz continuous if there exists a constant $\beta \geq 0$ such that

$$
\|T(x)-T(y)\| \leq \beta\|\eta(x, y)\| ;
$$

(xiii) $(\eta, \gamma)$-partially relaxed monotone if there exists a positive constant $\gamma$ such that

$$
\langle T(x)-T(y), \eta(z, y)\rangle \geq(-\gamma)\|z-x\|^{2} \quad \forall x, y, z \in H
$$

(xiv) $(\eta, \gamma)$-partially relaxed pseudomonotone if there exists a positive constant $\gamma$ such that

$$
\langle T(y), \eta(z, y)\rangle \geq 0 \Longrightarrow\langle T(x), \eta(z, y)\rangle \geq(-\gamma)\|z-x\|^{2} \quad \forall x, y, z \in H .
$$

Lemma 1.4. Let $T: H \rightarrow H$ be $(\eta, \alpha)$-cocoercive and let $\eta: H \times H \rightarrow H$ be a mapping such that

(i) $\|\eta(x, y)\| \leq \lambda\|x-y\|$;

(ii) $\eta(x, y)+\eta(y, x)=0$;

(iii) $\eta(x, y)=\eta(x, z)+\eta(z, y)$.

Then $T$ is $\left(\eta,-\left(\lambda^{2} / 4 \alpha\right)\right)$-partially relaxed monotone. 
4 Auxiliary problem principle

Proof. Since $T: H \rightarrow H$ is $(\eta, \alpha)$-cocoercive, we have

$$
\begin{aligned}
\langle T(x)-T(y), \eta(z, y)\rangle & =\langle T(x)-T(y), \eta(z, x)\rangle+\langle T(x)-T(y), \eta(x, y)\rangle \\
& \geq \alpha\|T(x)-T(y)\|^{2}+\langle T(x)-T(y), \eta(z, x)\rangle \\
& =\alpha\left(\|T(x)-T(y)\|^{2}+\frac{1}{\alpha}\langle T(x)-T(y), \eta(z, x)\rangle\right) \\
& \geq-\left\{\frac{1}{4 \alpha}\|\eta(z, x)\|^{2}\right\} \geq-\left\{\frac{\lambda^{2}}{4 \alpha}\|z-x\|^{2}\right\} .
\end{aligned}
$$

Definition 1.5. A mapping $T: H \rightarrow H$ is said to be $\mu$-cocoercive [2] if for each $x, y \in H$, there exists

$$
\langle T(x)-T(y), x-y\rangle \geq \mu\|T(x)-T(y)\|^{2},
$$

where $\mu$ is a positive constant.

Example 1.6. Let $T: K \rightarrow H$ be nonexpansive. Then $I-T$ is $1 / 2$-cocoercive, where $I$ is the identity mapping on $H$. For if $x, y \in K$, we have

$$
\begin{aligned}
\|(I-T)(x)-(I-T)(y)\|^{2} & =\|x-y-(T(x)-T(y))\|^{2} \\
& =\|x-y\|^{2}-2\langle x-y, T(x)-T(y)\rangle+\|T(x)-T(y)\|^{2} \\
& \leq 2\left\{\|x-y\|^{2}-\langle x-y, T(x)-T(y)\rangle\right\} \\
& =2\langle x-y,(I-T)(x)-(I-T)(y)\rangle,
\end{aligned}
$$

that is,

$$
\langle(I-T)(x)-(I-T)(y), x-y\rangle \geq \frac{1}{2}\|(I-T)(x)-(I-T)(y)\|^{2} .
$$

A subset $K$ of $H$ is said to be invex if there exists a function $\eta: K \times K \rightarrow H$ such that whenever $x, y \in K$ and $t \in[0,1]$, it follows that

$$
x+\operatorname{t\eta }(y, x) \in K
$$

A function $f: K \rightarrow R$ is called invex if whenever $x, y \in K$ and $t \in[0,1]$, it follows that

$$
f(x+t \eta(y, x)) \leq(1-t) f(x)+t f(y) .
$$

\section{Some auxiliary results}

This section deals with some auxiliary results [2] and their modified versions crucial to the approximation-solvability of VIP (1.1). Let $h: H \rightarrow R$ be a continuously Fréchetdifferentiable mapping on a Hilbert space $H$. It follows that $h^{\prime}(x) \in L(H, R)$ - the space of all bounded linear operators from $H$ into $R$. From now on, we will denote the real number $h^{\prime}(x)(y)$ by $\left\langle h^{\prime}(x), y\right\rangle$ for all $x, y \in H$. 
Lemma 2.1. Let $H$ be a real Hilbert space and let $K$ be a nonempty closed invex subset of $H$. Let $h^{\prime}$, the gradient of $h: K \rightarrow R$, be $(\eta, \alpha)$-strongly monotone on $K$ and let the following assumptions hold.

(i) There exist an $x^{*} \in K$ and a number $r \geq 0$ such that for all $x \in K^{*}$ and $t \in[0,1]$, the mapping $\eta: K \times K \rightarrow H$ satisfies

$$
\|\eta(x, y)\| \leq r
$$

(ii) The set $S^{*}$ defined by

$$
S^{*}=\left\{(h, \eta): h^{\prime}\left(x^{*}+t\left(x-x^{*}\right)\right)\left(x-x^{*}\right) \geq\left\langle h^{\prime}\left(x^{*}+t \eta\left(x, x^{*}\right)\right), \eta\left(x, x^{*}\right)\right\rangle\right\}
$$

is nonempty, where $h: K \rightarrow R$ is a continuously Fréchet-differentiable mapping, and the set $K^{*}$ is defined by

$$
K^{*}=\left\{x \in K:\left\|x-x^{*}\right\| \leq r\right\}
$$

Then for all $x \in K^{*}$ and $(h, \eta) \in S^{*}$,

$$
h(x)-h\left(x^{*}\right)-\left\langle h^{\prime}\left(x^{*}\right), \eta\left(x, x^{*}\right)\right\rangle \geq \frac{\alpha}{2}\left\|x-x^{*}\right\|^{2} .
$$

Lemma 2.2. Let $H$ be a real Hilbert space and let $K$ be a nonempty closed convex subset of $H$. Let $h^{\prime}$, the gradient of $h: K \rightarrow R$, be $(\alpha)$-strongly monotone on $K$ and let $h: K \rightarrow R$ be a continuously Fréchet-differentiable mapping. Then for all $x, x^{*} \in K$,

$$
h(x)-h\left(x^{*}\right)-\left\langle h^{\prime}\left(x^{*}\right), x-x^{*}\right\rangle \geq \frac{\alpha}{2}\left\|x-x^{*}\right\|^{2} .
$$

Lemma 2.3. Let $H$ be a real Hilbert space and let $K$ be a nonempty closed invex subset of $H$. Let $h^{\prime}$, the gradient of $h: K \rightarrow R$, be $(\eta, \delta)$-Lipschitz continuous on $K$ and let the following assumptions hold.

(i) There exist an $x^{*} \in K$ and a number $q \geq 0$ such that for all $x \in K_{1}$ and $t \in[0,1]$, the mapping $\eta: K \times K \rightarrow H$ satisfies

$$
\|\eta(x, y)\| \leq q
$$

(ii) The set $S_{1}$ defined by

$$
S_{1}=\left\{(h, \eta): h^{\prime}\left(x^{*}+t\left(x-x^{*}\right)\right)\left(x-x^{*}\right) \leq\left\langle h^{\prime}\left(x^{*}+t \eta\left(x, x^{*}\right)\right), \eta\left(x, x^{*}\right)\right\rangle\right\}
$$

is nonempty, where $h: K \rightarrow R$ is a continuously Fréchet-differentiable mapping, and the set $K_{1}$ is defined by

$$
K_{1}=\left\{x \in K:\left\|x-x^{*}\right\| \leq q\right\} .
$$

Then for all $x \in K_{1}$ and $(h, \eta) \in S_{1}$,

$$
h(x)-h\left(x^{*}\right)-\left\langle h^{\prime}\left(x^{*}\right), \eta\left(x, x^{*}\right)\right\rangle \leq \frac{\delta}{2}\left\|x-x^{*}\right\|^{2} .
$$




\section{General auxiliary problem principle}

In this section, we present the approximation-solvability of the VIP (1.1) using the convergence analysis for the general auxiliary problem principle.

Algorithm 3.1. For arbitrarily chosen initial point $x^{0} \in K$, determine an iterate $x^{k+1}$ such that

$$
\left\langle\rho T\left(x^{k}\right)+h^{\prime}\left(x^{k+1}\right)-h^{\prime}\left(x^{k}\right), \eta\left(x, x^{k+1}\right)\right\rangle+\rho\left(f(x)-f\left(x^{k+1}\right)\right) \geq 0,
$$

for all $x \in K$, where $h: K \rightarrow R$ is continuously Fréchet-differentiable, $f: K \rightarrow R$ is proper, invex, and lower semicontinuous, $\rho>0$, and $\eta: K \times K \rightarrow H$ is any mapping.

Algorithm 3.2. For arbitrarily chosen initial point $x^{0} \in K$, determine an iterate $x^{k+1}$ such that

$$
\left\langle\rho T\left(x^{k}\right)+h^{\prime}\left(x^{k+1}\right)-h^{\prime}\left(x^{k}\right), x-x^{k+1}\right\rangle+\rho\left(f(x)-f\left(x^{k+1}\right)\right) \geq 0,
$$

for all $x \in K$, where $h: K \rightarrow R$ is continuously Fréchet-differentiable, $\rho>0$, and $K$ is $a$ nonempty closed convex subset of $H$.

Algorithm 3.3. For arbitrarily chosen initial point $x^{0} \in K$, determine an iterate $x^{k+1}$ such that

$$
\left\langle\rho T\left(x^{k}\right)+h^{\prime}\left(x^{k+1}\right)-h^{\prime}\left(x^{k}\right), x-x^{k+1}\right\rangle \geq 0,
$$

for all $x \in K$, where $h: K \rightarrow R$ is continuously Fréchet-differentiable, $\rho>0$, and $K$ is $a$ nonempty closed convex subset of $H$.

We now present, based on Algorithm 3.1, the approximation solvability of the VIP (1.1) in a Hilbert space setting.

Theorem 3.4. Let $H$ be a real infinite-dimensional Hilbert space and let $K$ be a nonempty closed invex subset of $H$. Let $T: K \rightarrow H$ be $(\eta, \gamma)$-partially relaxed pseudomonotone. Let $f: K \rightarrow R$ be proper, invex, and lower semicontinuous on $K$, let $h: K \rightarrow R$ be continuously Fréchet-differentiable on $K$ with $h^{\prime}$, the gradient of $h,(\eta, \alpha)$-strongly monotone, and $(\eta, \beta)$ Lipschitz continuous, and let $h^{\prime}$ be continuous from $H$ equipped with weak topology to $H$ equipped with strong topology. Suppose that the following assumptions hold.

(i) There exist $a y^{*} \in K$ and numbers $a>0, r \geq 0, \rho(a<\rho<\alpha / 2 \gamma)$ such that for all $t \in[0,1]$ and for all $x \in K^{*}$, the set $S^{*}$ defined by

$$
S^{*}=\left\{(h, \eta): h^{\prime}\left(y^{*}+t\left(x-y^{*}\right)\right)\left(x-y^{*}\right) \geq\left\langle h^{\prime}\left(y^{*}+t \eta\left(x, y^{*}\right)\right), \eta\left(x, y^{*}\right)\right\rangle\right\}
$$

is nonempty, where

$$
K^{*}=\left\{x \in K:\left\|x-y^{*}\right\| \leq r\right\} \subset K .
$$

(ii) The mapping $\eta: K \times K \rightarrow H$ is $(\lambda)$-Lipschitz continuous.

(iii) $\eta(u, v)+\eta(v, u)=0$ and $\eta(u, v)=\eta(u \cdot w)+\eta(w, v)$. 
(iv) For each fixed $y \in K$, the map $x \rightarrow \eta(y, x)$ is sequentially continuous from the weak topology to the weak topology.

(v) $\|\eta(u, v)\| \leq r$.

Then an iterate $x^{k+1}$ is a unique solution to (3.1).

If, in addition, $x^{*} \in K$ is a solution to VIP (1.1) and $\left\|T\left(x^{k}\right)-T\left(x^{*}\right)\right\| \rightarrow 0$, then the sequence $\left\{x^{k}\right\}$ generated by Algorithm 3.1 converges weakly to $x^{*}$.

Proof. First to show that $x^{k+1}$ is a unique solution to (3.1), assume that $y^{k+1}$ is another distinct solution to (3.1). Since $h^{\prime}$ is $(\eta, \alpha)$-strongly monotone, it follows applying (3.1) that

$$
-\left\langle h^{\prime}\left(x^{k+1}\right)-h^{\prime}\left(y^{k+1}\right), \eta\left(x^{k+1}, y^{k+1}\right)\right\rangle \geq 0,
$$

or

$$
\left\|x^{k+1}-y^{k+1}\right\|^{2} \leq 0
$$

a contradiction.

Since $x^{*} \in K$ is a solution to the VIP (1.1), we define a function $\Delta^{*}$ by

$$
\Delta^{*}(x):=h\left(x^{*}\right)-h(x)-\left\langle h^{\prime}(x), \eta\left(x^{*}, x\right)\right\rangle .
$$

Then applying Lemma 2.1, we have

$$
\Delta^{*}(x):=h\left(x^{*}\right)-h(x)-\left\langle h^{\prime}(x), \eta\left(x^{*}, x\right)\right\rangle \geq \frac{\alpha}{2}\left\|x^{*}-x\right\|^{2} .
$$

It follows that

$$
\Delta^{*}\left(x^{k+1}\right):=h\left(x^{*}\right)-h\left(x^{k+1}\right)-\left\langle h^{\prime}\left(x^{k+1}\right), \eta\left(x^{*}, x^{k+1}\right)\right\rangle .
$$

Now we can write

$$
\begin{aligned}
\Delta^{*}\left(x^{k}\right)-\Delta^{*}\left(x^{k+1}\right)= & h\left(x^{k+1}\right)-h\left(x^{k}\right)-\left\langle h^{\prime}\left(x^{k}\right), \eta\left(x^{k+1}, x^{k}\right)\right\rangle \\
& +\left\langle h^{\prime}\left(x^{k+1}\right)-h^{\prime}\left(x^{k}\right), \eta\left(x^{*}, x^{k+1}\right)\right\rangle \\
\geq & \frac{\alpha}{2}\left\|x^{k+1}-x^{k}\right\|^{2}+\left\langle h^{\prime}\left(x^{k+1}\right)-h^{\prime}\left(x^{k}\right), \eta\left(x^{*}, x^{k+1}\right)\right\rangle \\
\geq & \frac{\alpha}{2}\left\|x^{k+1}-x^{k}\right\|^{2}+\rho\left\langle T\left(x^{k}\right), \eta\left(x^{k+1}, x^{*}\right)\right\rangle \\
& +\rho\left(f\left(x^{k+1}\right)-f\left(x^{*}\right)\right),
\end{aligned}
$$

for $x=x^{*}$ in (3.1).

Therefore, we have

$$
\Delta^{*}\left(x^{k}\right)-\Delta^{*}\left(x^{k+1}\right) \geq \frac{\alpha}{2}\left\|x^{k+1}-x^{k}\right\|^{2}+\rho\left\langle T\left(x^{k}\right), \eta\left(x^{k+1}, x^{*}\right)\right\rangle+\rho\left(f\left(x^{k+1}\right)-f\left(x^{*}\right)\right) .
$$

If we replace $x$ by $x^{k+1}$ in (1.1), we obtain

$$
\left\langle T\left(x^{*}\right), \eta\left(x^{k+1}, x^{*}\right)\right\rangle+f\left(x^{k+1}\right)-f\left(x^{*}\right) \geq 0 .
$$


Since $T$ is $(\eta, \gamma)$-partially relaxed pseudomonotone, it implies in light of (3.13) that

$$
\Delta^{*}\left(x^{k}\right)-\Delta^{*}\left(x^{k+1}\right) \geq \frac{\alpha}{2}\left\|x^{k+1}-x^{k}\right\|^{2}-\rho \gamma\left\|x^{k+1}-x^{k}\right\|^{2}=\left(\frac{\alpha}{2}-\rho \gamma\right)\left\|x^{k+1}-x^{k}\right\|^{2}
$$

for $\rho<(\alpha / 2 \gamma)$.

It follows that the sequence $\left\{\Delta^{*}\left(x^{k}\right)\right\}$ is a strictly decreasing sequence except for $x^{k+1}=$ $x^{k}$, and in that situation $x^{k}$ is a solution to (1.1). Since the difference of two consecutive terms tends to zero as $k \rightarrow \infty$, it implies that

$$
\left\|x^{k+1}-x^{k}\right\| \longrightarrow 0 \quad \text { as } k \longrightarrow \infty
$$

On the top of that, in light of Lemma 2.1, we have

$$
\left\|x^{*}-x^{k}\right\|^{2} \leq \frac{2}{\alpha} \Delta^{*}\left(x^{k}\right)
$$

and so the sequence $\left\{x^{k}\right\}$ is bounded. Let $x^{\prime}$ be a cluster point of the sequence $\left\{x^{k}\right\}$, that is, there exists a subsequence $\left\{x^{k j}\right\}$ of the sequence $\left\{x^{k}\right\}$ such that $\left\{x^{k j}\right\}$ converges weakly to $x^{\prime}$. Since $h^{\prime}$ is $(\eta, \beta)$-Lipschitz continuous and $a<\rho$, it follows using (3.1) that for some $x \in K$, we have

$$
\begin{aligned}
\left\langle\rho T\left(x^{k}\right), \eta\left(x, x^{k+1}\right)\right\rangle+\rho\left(f(x)-f\left(x^{k+1}\right)\right) & \geq-\left\langle h^{\prime}\left(x^{k+1}\right)-h^{\prime}\left(x^{k}\right), \eta\left(x, x^{k+1}\right)\right\rangle \\
& \geq-\beta\left\|x^{k+1}-x^{k}\right\|\left\|\eta\left(x, x^{k+1}\right)\right\|,
\end{aligned}
$$

or

$$
\left\langle T\left(x^{k}\right), \eta\left(x, x^{k+1}\right)\right\rangle+f(x)-f\left(x^{k+1}\right) \geq-\frac{\beta}{a}\left\|x^{k+1}-x^{k}\right\|\left\|\eta\left(x, x^{k+1}\right)\right\|
$$

Since $T\left(x^{k j}\right)$ converges strongly to $T\left(x^{*}\right)$ and $\left\|x^{k j+1}-x^{k j}\right\| \rightarrow 0$, and $f$ is invex and lower semicontinuous (and hence $f$ is weakly lower semicontinuous), it follows from (3.18) that

$$
\left\langle T\left(x^{*}\right), \eta\left(x, x^{\prime}\right)\right\rangle+f(x)-f\left(x^{\prime}\right) \geq 0 \quad \forall x \in K,
$$

while

$$
\begin{gathered}
\left\langle T\left(x^{\prime}\right), \eta\left(x^{k j}, x^{\prime}\right)\right\rangle+f(x)-f\left(x^{k j}\right) \longrightarrow 0 \\
\left\langle T\left(x^{\prime}\right), \eta\left(x^{k j}, x^{\prime}\right)\right\rangle \longrightarrow 0 .
\end{gathered}
$$

At this stage, if $T\left(x^{\prime}\right)=0$, then $x^{\prime}$ is a solution to the $\operatorname{VIP}(1.1)$; and if $T\left(x^{\prime}\right) \neq 0$, then we express it in the form

$$
\eta\left(y^{k j}, x^{k j}\right)=-\frac{\left\langle T\left(x^{\prime}\right), \eta\left(x^{k j}, x^{\prime}\right)\right\rangle T\left(x^{\prime}\right)}{\left\|T\left(x^{\prime}\right)\right\|^{2}}
$$


It follows that

$$
\left\langle T\left(x^{\prime}\right), \eta\left(y^{k j}, x^{\prime}\right)\right\rangle=0
$$

and thus, we have

$$
\left\|\eta\left(y^{k j}, x^{k j}\right)\right\| \longrightarrow 0
$$

It follows that

$$
y^{k j}-x^{\prime}
$$

Applying (3.22), we have

$$
0=\left\langle T\left(x^{\prime}\right), \eta\left(y^{k j}, x^{\prime}\right)\right\rangle=\left\langle T\left(x^{\prime}\right), \eta\left(y^{k j}, x^{*}\right)\right\rangle+\left\langle T\left(x^{\prime}\right), \eta\left(x^{*}, x^{\prime}\right)\right\rangle .
$$

Since $T\left(x^{\prime}\right) \neq 0$, it follows that $y^{k j} \rightarrow x^{*}$ and $x^{*}=x^{\prime}$, a solution to the VIP (1.1).

Corollary 3.5. Let $H$ be a real infinite-dimensional Hilbert space and let $K$ be a nonempty closed invex subset of $H$. Let $T: K \rightarrow H$ be $(\eta, \gamma)$-pseudococoercive. Let $f: K \rightarrow R$ be proper, invex, and lower semicontinuous on $K$, let $h: K \rightarrow R$ be continuously Fréchet-differentiable on $K$ with $h^{\prime}$, the gradient of $h,(\eta, \alpha)$-strongly monotone and $(\eta, \beta)$-Lipschitz continuous, and let $h^{\prime}$ be continuous from $H$ equipped with weak topology to $H$ equipped with strong topology. Suppose that the following assumptions hold.

(i) There exist $a y^{*} \in K$ and numbers $a>0, r \geq 0, \rho(a<\rho<\alpha / 2 \gamma)$ such that for all $t \in[0,1]$ and for all $x \in K^{*}$, the set $S^{*}$ defined by

$$
S^{*}=\left\{(h, \eta): h^{\prime}\left(y^{*}+t\left(x-y^{*}\right)\right)\left(x-y^{*}\right) \geq\left\langle h^{\prime}\left(y^{*}+t \eta\left(x, y^{*}\right)\right), \eta\left(x, y^{*}\right)\right\rangle\right\}
$$

is nonempty, where

$$
K^{*}=\left\{x \in K:\left\|x-y^{*}\right\| \leq r\right\} \subset K .
$$

(ii) The mapping $\eta: K \times K \rightarrow H$ is $(\lambda)$-Lipschitz continuous.

(iii) $\eta(u, v)+\eta(v, u)=0$ and $\eta(u, v)=\eta(u \cdot w)+\eta(w, v)$.

(iv) For each fixed $y \in K$, the map $x \rightarrow \eta(y, x)$ is sequentially continuous from the weak topology to the weak topology.

(v) $\|\eta(u, v)\| \leq r$.

Then an iterate $x^{k+1}$ is a unique solution to (3.1).

If $x^{*} \in K$ is a solution to VIP (1.1), then the sequence $\left\{x^{k}\right\}$ generated by Algorithm 3.1 converges weakly to $x^{*}$.

For $f=0$ and $\eta(u, v)=u-v$ in Corollary 3.5, it reduces to the following corollary.

Corollary 3.6. Let $H$ be a real infinite-dimensional Hilbert space and let $K$ be a nonempty closed convex subset of $H$. Let $T: K \rightarrow H$ be $(\gamma)$-pseudococoercive. Let $h: K \rightarrow R$ be continuously Fréchet-differentiable on $K$ with $h^{\prime}$, the gradient of $h,(\alpha)$-strongly monotone, and $(\beta)$-Lipschitz continuous, and let $h^{\prime}$ be continuous from $H$ equipped with weak topology to $H$ equipped with strong topology. Then an iterate $x^{k+1}$ is a unique solution to (3.3). If $x^{*} \in K$ is 
a solution to VIP (1.3), then the sequence $\left\{x^{k}\right\}$ generated by Algorithm 3.3 converges weakly to $x^{*}$.

Note that Corollary 3.6 is proved in [7, Theorem 4.1] with an additional imposition of the uniform continuity on the mapping $T$, but we feel that the uniform continuity is not required for the convergence purposes.

Theorem 3.7. Let $H$ be a real infinite-dimensional Hilbert space and let $K$ be a nonempty closed invex subset of $H$. Let $T: K \rightarrow H$ be $(\eta, \gamma)$-partially relaxed pseudomonotone. Let $f: K \rightarrow R$ be proper, invex, and lower semicontinuous on $K$, let $h: K \rightarrow R$ be continuously Fréchet-differentiable on $K$ with $h^{\prime}$, the gradient of $h,(\eta, \alpha)$-strongly monotone, and $(\eta, \beta)$ Lipschitz continuous, and let $h^{\prime}$ be continuous from $H$ equipped with weak topology to $H$ equipped with strong topology. Suppose that the following assumptions hold.

(i) There exist $a y^{*} \in K$ and numbers $a>0, r \geq 0, q \geq 0, \rho(a<\rho<\alpha / 2 \gamma)$ such that for all $t \in[0,1]$ and for all $x \in K^{*}$, the set $S^{*}$ defined by

$$
S^{*}=\left\{(h, \eta): h^{\prime}\left(y^{*}+t\left(x-y^{*}\right)\right)\left(x-y^{*}\right) \geq\left\langle h^{\prime}\left(y^{*}+t \eta\left(x, y^{*}\right)\right), \eta\left(x, y^{*}\right)\right\rangle\right\}
$$

is nonempty, where

$$
K^{*}=\left\{x \in K:\left\|x-y^{*}\right\| \leq r\right\} \subset K .
$$

(ii) The mapping $\eta: K \times K \rightarrow H$ is $(\lambda)$-Lipschitz continuous.

(iii) $\eta(u, v)+\eta(v, u)=0$ and $\eta(u, v)=\eta(u \cdot w)+\eta(w, v)$.

(iv) For each fixed $y \in K$ the map $x \rightarrow \eta(y, x)$ is sequentially continuous from the weak topology to the weak topology.

(v) $\|\eta(u, v)\| \leq r$.

Then an iterate $x^{k+1}$ is a unique solution to (3.1). If $x^{*} \in K$ is a solution to VIP (1.1) and $\left\|T\left(x^{k}\right)-T\left(x^{*}\right)\right\| \rightarrow 0$, then the sequence $\left\{x^{k}\right\}$ generated by Algorithm 3.1 converges weakly to $x^{*}$.

In addition, assume that

(vi) there exist a $y^{*} \in K$ such that for all $x \in K_{1}$, the set $S_{1}$ defined by

$$
S_{1}=\left\{(h, \eta): h^{\prime}\left(y^{*}+t\left(x-y^{*}\right)\right)\left(x-y^{*}\right) \leq\left\langle h^{\prime}\left(y^{*}+t \eta\left(x, y^{*}\right)\right), \eta\left(x, y^{*}\right)\right\rangle\right\}
$$

is nonempty, where

$$
K_{1}=\left\{x \in K:\left\|x-y^{*}\right\| \leq q\right\} \subset K,
$$

with $\left\|\eta\left(x, y^{*}\right)\right\| \leq q$.

Then the sequence $\left\{x^{k}\right\}$ generated by Algorithm 3.1 converges to $x^{*}$.

Proof. Since based on the proof of Theorem 3.4, $x^{\prime}$ is a weak cluster point of the sequence $\left\{x^{k}\right\}$, we define a function $\Lambda^{*}$ by

$$
\Lambda^{*}\left(x^{k}\right)=h\left(x^{\prime}\right)-h\left(x^{k}\right)-\left\langle h^{\prime}\left(x^{k}\right), \eta\left(x^{\prime}, x^{k}\right)\right\rangle .
$$


Applying Lemmas 2.1 and 2.3, we have the following:

$$
\begin{aligned}
& \Lambda^{*}\left(x^{k}\right)=h\left(x^{\prime}\right)-h\left(x^{k}\right)-\left\langle h^{\prime}\left(x^{k}\right), \eta\left(x^{\prime}, x^{k}\right)\right\rangle \geq \frac{\alpha}{2}\left\|x^{\prime}-x^{k}\right\|^{2} . \\
& \Lambda^{*}\left(x^{k}\right)=h\left(x^{\prime}\right)-h\left(x^{k}\right)-\left\langle h^{\prime}\left(x^{k}\right), \eta\left(x^{\prime}, x^{k}\right)\right\rangle \leq \frac{\beta}{2}\left\|x^{\prime}-x^{k}\right\|^{2} .
\end{aligned}
$$

It follows from (3.34) that

$$
\lim _{n \rightarrow \infty} \Lambda^{*}\left(x^{k}\right)=0
$$

Applying (3.35) to (3.33), it follows that the entire sequence $\left\{x^{k}\right\}$ generated by Algorithm 3.1 converges to $x^{\prime}$.

\section{References}

[1] I. K. Argyros and R. U. Verma, On general auxiliary problem principle and nonlinear mixed variational inequalities, Nonlinear Functional Analysis and Applications 6 (2001), no. 2, 247-256.

[2] __ Generalized partial relaxed monotonicity and solvability of nonlinear variational inequalities, Panamerican Mathematical Journal 12 (2002), no. 3, 85-104.

[3] G. Cohen, Auxiliary problem principle and decomposition of optimization problems, Journal of Optimization Theory and Applications 32 (1980), no. 3, 277-305.

[4] __ Auxiliary problem principle extended to variational inequalities, Journal of Optimization Theory and Applications 59 (1988), no. 2, 325-333.

[5] J. Eckstein, Nonlinear proximal point algorithms using Bregman functions, with applications to convex programming, Mathematics of Operations Research 18 (1993), no. 1, 202-226.

[6] J. Eckstein and D. P. Bertsekas, On the Douglas-Rachford splitting method and the proximal point algorithm for maximal monotone operators, Mathematical Programming Series A 55 (1992), no. 3, 293-318.

[7] N. El Farouq, Pseudomonotone variational inequalities: convergence of the auxiliary problem method, Journal of Optimization Theory and Applications 111 (2001), no. 2, 305-326.

[8] _ Pseudomonotone variational inequalities: convergence of proximal methods, Journal of Optimization Theory and Applications 109 (2001), no. 2, 311-326.

[9] S. Karamardian, Complementarity problems over cones with monotone and pseudomonotone maps, Journal of Optimization Theory and Applications 18 (1976), no. 4, 445-454.

[10] S. Karamardian and S. Schaible, Seven kinds of monotone maps, Journal of Optimization Theory and Applications 66 (1990), no. 1, 37-46.

[11] B. Martinet, Régularisation d'inéquations variationnelles par approximations successives, Revue Française d'Informatique Recherche Opérationnelle 4 (1970), Ser. R-3, 154-158 (French).

[12] Z. Naniewicz and P. D. Panagiotopoulos, Mathematical theory of hemivariational inequalities and applications, Monographs and Textbooks in Pure and Applied Mathematics, vol. 188, Marcel Dekker, New York, 1995.

[13] R. T. Rockafellar, Augmented Lagrangians and applications of the proximal point algorithm in convex programming, Mathematics of Operations Research 1 (1976), no. 2, 97-116.

[14] Monotone operators and the proximal point algorithm, SIAM Journal Control Optimization 14 (1976), no. 5, 877-898.

[15] R. U. Verma, Nonlinear variational and constrained hemivariational inequalities involving relaxed operators, Zeitschrift für Angewandte Mathematik und Mechanik 77 (1997), no. 5, 387-391.

[16] _ Approximation-solvability of nonlinear variational inequalities involving partially relaxed monotone (PRM) mappings, Advances in Nonlinear Variational Inequalities 2 (1999), no. 2, 137148. 


\section{Auxiliary problem principle}

[17] _ A new class of iterative algorithms for approximation-solvability of nonlinear variational inequalities, Computers \& Mathematics with Applications 41 (2001), no. 3-4, 505-512.

[18] _ General auxiliary problem principle involving multivalued mappings, Nonlinear Functional Analysis and Applications 8 (2003), no. 1, 105-110.

[19] _ Generalized strongly nonlinear variational inequalities, Revue Roumaine de Mathématiques Pures et Appliquées. Romanian Journal of Pure and Applied Mathematics 48 (2003), no. 4, 431-434.

[20] _ Nonlinear implicit variational inequalities involving partially relaxed pseudomonotone mappings, Computers \& Mathematics with Applications 46 (2003), no. 10-11, 1703-1709.

[21] _ Partial relaxed monotonicity and general auxiliary problem principle with applications, Applied Mathematics Letters 16 (2003), no. 5, 791-796.

[22] _ Partially relaxed cocoercive variational inequalities and auxiliary problem principle, Journal of Applied Mathematics and Stochastic Analysis 2004 (2004), no. 2, 143-148.

[23] E. Zeidler, Nonlinear Functional Analysis and Its Applications. II/B. Nonlinear Monotone Operators, Springer, New York, 1990.

Ram U. Verma: Department of Mathematics, University of Toledo, Toledo, OH 43606, USA

E-mail address: verma99@msn.com 